\title{
Strain-dependent induction of epithelial cell oncosis by Campylobacter jejuni is correlated with invasion ability and is independent of cytolethal distending toxin
}

Correspondence
Andre G. Buret
aburet@ucalgary.ca

Received 31 October 2006

Revised 15 May 2007

Accepted 17 May 2007
Lisa D. Kalischuk, ${ }^{1,2}$ G. Douglas Inglis ${ }^{2}$ and Andre G. Buret $^{1}$

\author{
${ }^{1}$ Department of Biological Sciences, Inflammation Research Network, University of Calgary, \\ Bio 336, 2500 University Drive NW, Calgary, AB T2N 1N4, Canada \\ ${ }^{2}$ Agriculture and Agri-Food Canada, 5403-1st Avenue S, Lethbridge, AB T1J 4B1, Canada
}

\begin{abstract}
Induction of host cell death is thought to play an important role in bacterial pathogenesis. Campylobacter jejuni is a prevalent cause of bacterial enteritis; however, its effects on enterocytes remain unclear. The present study indicates for the first time that $C$. jejuni induces oncotic, rather than apoptotic death of T84 enterocytes. C. jejuni-treated enterocytes exhibited extensive cytoplasmic vacuolation, rapid (3-6 h) loss of plasma membrane integrity ('cytotoxicity'), loss of mitochondrial transmembrane potential, and ATP depletion. Enterocytes also exhibited increased oligonucleosomal DNA fragmentation, a feature characteristic of apoptosis. However, consistent with a non-apoptotic process, DNA fragmentation and cytotoxicity were not caspase dependent. During apoptosis, caspases mediate cleavage of poly(ADP-ribose) polymerase; however, cleavage was not observed in C. jejuni-treated monolayers. Cytotoxicity, ATP depletion and DNA fragmentation were not prevented by the deletion of the cytolethal distending toxin (CDT) gene, indicating that $C$. jejuni causes enterocyte oncosis via a mechanism that is CDT independent. The ability to cause oncosis was significantly decreased in a FlaAFlaB mutant $\left(\mathrm{CDT}^{+}\right)$that was defective in the ability to adhere and invade enterocytes. Analysis of clinical isolates revealed that oncosis was strain dependent and correlated with increased invasive ability. These observations offer new insights into the pathogenesis of $C$. jejuni infection.
\end{abstract}

\section{INTRODUCTION}

Campylobacter jejuni is the most prevalent cause of human bacterial enteritis in North America [Centers for Disease Control and Prevention (http://www.cdc.gov/foodnet/); Health Canada (http://dsol-smed.hc-sc.gc.ca/dsol-med/ ndis/cdise.html)]. Infected humans exhibit a range of symptoms varying from mild watery diarrhoea to severe inflammatory diarrhoea often containing blood and leukocytes (Wassenaar \& Blaser, 1999). Histological examination of affected intestinal tissues typically reveals necrotic ulceration of the mucosal epithelium with crypt abscesses and inflammatory infiltration of neutrophils into the lamina propria (Wassenaar \& Blaser, 1999). Tissue damage appears to be largely due to the effects of cytotoxins (Wassenaar, 1997) and/or host-cell invasion (Everest et al., 1992; Russell et al., 1993). Although the pathological features of campylobacteriosis have been well described, the host-pathogen interactions involved in inciting inflammation remain poorly understood.

Abbreviations: CDT, cytolethal distending toxin; LDH, lactate dehydrogenase; PARP, poly(ADP-ribose) polymerase.
Host cell death is conventionally divided into the morphologically and biochemically distinct processes of apoptosis or oncosis (Majno \& Joris, 1995). Apoptosis is a genetically regulated, energy-dependent process that plays a critical role in the elimination of cells during development and homeostasis (Ren \& Savill, 1998), but is also implicated in the pathogenesis of a variety of enteric micro-organisms (Chin et al., 2002; Fiorentini et al., 1998; Kim et al., 1998; Zychlinsky, 1993). Excessive enterocyte death may ultimately disrupt the intestinal epithelial barrier, allowing for increased pathogen translocation and dissemination (Buret et al., 2003; Chin et al., 2002). Apoptosis is characterized by chromatin condensation and fragmentation, membrane blebbing and formation of apoptotic bodies with intact plasma membranes (Majno \& Joris, 1995). Phagocytic disposal of the apoptotic bodies prevents release of cellular debris and subsequent inflammation (Ren \& Savill, 1998). In contrast, oncosis (often referred to as 'necrosis') is a rapid process characterized by vacuolation, mitochondrial swelling and loss of plasma membrane integrity (Majno \& Joris, 1995). The resultant leakage of cellular content into the intercellular milieu 
often incites an inflammatory response leading to tissue injury (Ren \& Savill, 1998).

Several morphological features can be used to distinguish apoptotic from oncotic cells (Majno \& Joris, 1995). During oncosis, cells rapidly lose plasma membrane integrity after the death stimulus whereas during apoptosis, plasma membrane integrity is maintained throughout the death process until the late onset of secondary necrosis. Caspases, particularly caspase-3, play a crucial role in apoptosis by effecting many of the characteristics of apoptosis (Earnshaw et al., 1999). Oligonuclosomal DNA fragmentation, which is considered a hallmark of apoptosis, is initiated by caspase-activated deoxyribonulclease (CAD) upon caspase-3 cleavage of its inhibitor, ICAD (Enari et al., 1998). During apoptosis, caspase-3 also cleaves the DNA repair enzyme poly(ADP-ribose) polymerase (PARP). Inactivation of PARP prevents DNA repair and conserves cellular ATP, which is required to complete the apoptotic process (Los et al., 2002).

Cell viability is largely regulated at the level of mitochondria, and cell death may be initiated by factors that cause depolarization of the mitochondrial membrane. Progression to apoptotic or oncotic cell death depends in part on the effect that mitochondrial transmembrane depolarization has on cellular ATP levels (Kim et al., 2003). Cellular ATP plays a pivotal role in determining whether cell death will be apoptotic or oncotic in nature (Eguchi et al., 1997; Leist et al., 1997). Apoptosis is an active energy-dependent process. Specifically, ATP is required for the formation of the apoptosome, which is involved in initiating the intrinsic apoptotic death cascade (Li et al., 1997). If damage to the mitochondria is such that ATP levels are insufficient to complete the apoptotic process, the mode of death may be redirected towards oncosis (Eguchi et al., 1997; Leist et al., 1997).

The cytotoxic effects of Campylobacter have been ascribed to the actions of several different toxins. However, to date only the cytolethal distending toxin (CDT) has been identified through genome sequence analysis. CDT is a DNase-like toxin produced by several species of bacteria including Campylobacter spp. (Pickett et al., 1996). This holotoxin is composed of three subunits: $\mathrm{CdtB}$ has homology to the family of DNase I-like nucleases and is the active subunit, and the CdtA and $\mathrm{C}$ subunits function in the delivery of CdtB into target cells (Lara-Tejero \& Galan, 2001). CDT is translocated into the nucleus, where it induces double-stranded DNA damage. This activates the G1 and/or G2/M checkpoint response that results in cell cycle arrest, and ultimately leads to cell death via mechanisms that are poorly understood (Whitehouse et al., 1998). It has been postulated that CDT plays a role in the elimination of immune cells and therefore modulates the host immune response. The effects of CDT have primarily been described for lymphocytes and monocytes, in which it generally induces apoptosis (Hickey et al., 2005; Siegesmund et al., 2004; Zhu et al., 1999). However, CDT has also been shown recently to induce non-apoptotic death of endothelial cells (Bielaszewska et al., 2005). The mechanisms responsible for $C$. jejuni-induced enterocyte death remain obscure.

A prominent feature of campylobacteriosis is acute inflammation of the intestinal mucosa. As the mode of cell death is a decisive factor in the host inflammatory response, the present study characterized the nature of $C$. jejuni-induced enterocyte death and assessed the role of CDT in this process. Further experiments sought to determine whether the induction of enterocyte death corresponded with the ability of $C$. jejuni to invade enterocytes.

\section{METHODS}

Epithelial cell culture. Studies were performed using a human colonic epithelial cell line with a crypt-like phenotype, T84 cells (American Type Culture Collection). T84 cells were grown in a $1: 1$ mixture of DMEM and Ham's F-12 medium supplemented with $10 \%$ heat-inactivated fetal bovine serum (Sigma-Aldrich) containing $200 \mathrm{mM}$ L-glutamine, $100 \mathrm{U}$ penicillin $\mathrm{ml}^{-1}, 100 \mu \mathrm{g}$ streptomycin $\mathrm{ml}^{-1}$ and $80 \mu \mathrm{g}$ tylosin $\mathrm{ml}^{-1}$ (all from Sigma). Cells were incubated at $37{ }^{\circ} \mathrm{C}$ in a humidified atmosphere containing $\sim 5 \% \mathrm{CO}_{2}$. Medium was replenished every 2-3 days and confluent monolayers were passaged with $2 \times$ trypsin-EDTA (Invitrogen). Trypsinized cells were seeded at a density of $2 \times 10^{5}$ cells $\mathrm{ml}^{-1}$ into Lab-Tek chamber slides (400 $\mu$ l per well, Nalgene Nunc International) and six-well $(3 \mathrm{ml})$ or 48 -well $(400 \mu \mathrm{l})$ tissue-culture-treated plates (Costar). Unless noted otherwise, cells were grown to confluence prior to inoculation. Cells were used for experiments between passages 5 and 15 .

Bacterial strains and culture conditions. C. jejuni strain 81-176, a strain widely used in pathogenesis studies and originally isolated from an outbreak of diarrhoea associated with ingestion of raw bovine milk, was used throughout the study (Korlath et al., 1985). C. jejuni strain NCTC 11168 (clinical isolate) was also used as necessary (Gaynor et al., 2004). Additional C. jejuni strains included 23 clinical isolates obtained from patients residing within the Chinook Health Region of Southwestern Alberta who were suffering from enteritis. All isolates were presumptively identified as $C$. jejuni based on a positive hippurate test, and their identities were confirmed by PCR detection of the mapA gene, which is present only in C. jejuni (Denis et al., 1999). Isolates were stored in Brucella broth (Difco) containing $30 \%$ $(\mathrm{v} / \mathrm{v})$ glycerol at $-80{ }^{\circ} \mathrm{C}$. Prior to use, cultures were streaked onto Karmali agar (Oxoid) and grown microaerophilically $\left(5 \% \mathrm{O}_{2}, 10 \%\right.$ $\mathrm{CO}_{2}, 2 \% \mathrm{H}_{2}$ and $83 \% \mathrm{~N}_{2}$ ) at $37{ }^{\circ} \mathrm{C}$. Inoculum was prepared by growing C. jejuni in Casamino yeast extract (CYE) broth (Stanfield et al., 1987) containing, per litre, $30 \mathrm{~g}$ Casamino acids (Difco), $4 \mathrm{~g}$ yeast extract (Difco), $0.5 \mathrm{~g} \mathrm{KH}_{2} \mathrm{PO}_{4}, 10 \mathrm{mg} \mathrm{FeSO}$ and $2 \mathrm{~g}$ porcine gastric mucin (Sigma). Mucin has previously been shown to increase invasion of Hep-2 cells (De Melo \& Pechere, 1988). C. jejuni was grown for $14-16 \mathrm{~h}$ at $37^{\circ} \mathrm{C}$ at 100 r.p.m. in microaerophilic conditions. Bacteria were enumerated by plating tenfold serial dilutions onto Karmali agar.

Construction and characterization of isogenic CDT mutant. A mutant of $C$. jejuni 81-176 was constructed by deleting the entire $c d t B$ gene and part of the $c d t A C$ genes, and inserting a kanamycinresistance cassette into the deletion site. Briefly, this mutant was constructed by amplifying the promoterless $c d t A B C$ operon from $C$. jejuni 81-176 using previously described primers P8 and P9 (Purdy et al., 2000). All PCR reactions were carried out in $20 \mu \mathrm{l}$ volumes and 
contained $1 \times$ reaction buffer, $0.2 \mathrm{mM}$ dNTPs, $2 \mathrm{mM} \mathrm{MgCl}_{2}, 0.5 \mu \mathrm{M}$ of each primer and $1 \mathrm{U}$ HotStar Taq polymerase (Qiagen). The conditions for amplification were 1 cycle at $95{ }^{\circ} \mathrm{C}$ for $15 \mathrm{~min}$ followed by 30 cycles of $94{ }^{\circ} \mathrm{C}$ for $30 \mathrm{~s}, 45^{\circ} \mathrm{C}$ for $30 \mathrm{~s}, 72{ }^{\circ} \mathrm{C}$ for $2 \mathrm{~min}$ and a final extension of $10 \mathrm{~min}$ at $72{ }^{\circ} \mathrm{C}$. The resulting fragment was ligated with the pGEM-T Easy vector (Promega) and used to transform Escherichia coli GM2163 (Dam $\left.{ }^{-}\right)$. Mutants were selected on Luria-Bertani agar (LB) containing ampicillin $\left(100 \mu \mathrm{g} \mathrm{ml}^{-1}\right)$. Plasmid DNA was extracted with the QIAprep Spin Miniprep kit (Qiagen) and digested with BclI (New England Biolabs, NEB) to generate the deletion.

A 987 bp fragment of pACYC177 containing the Tn903 kanamycinresistance cassette $\left[a p h\left(3^{\prime}\right)-1 \mathrm{a}\left(\mathrm{Kn}^{\mathrm{R}}\right)\right]$ was amplified by PCR using primers F2kanbamH1 (5'-TTGTGGATCCTCAACAAAGCCACGTTGTGT-3') and R2kanbamH1 (5'-TTGTGGATCCTCCCGTCAAGTCAGCGTAAT-3') (BamHI restriction site underlined). The conditions for amplification were 1 cycle at $95{ }^{\circ} \mathrm{C}$ for $15 \mathrm{~min}$ followed by 30 cycles of $94{ }^{\circ} \mathrm{C}$ for $30 \mathrm{~s}, 50{ }^{\circ} \mathrm{C}$ for $30 \mathrm{~s}, 72{ }^{\circ} \mathrm{C}$ for $1.5 \mathrm{~min}$ and a final extension of $10 \mathrm{~min}$ at $72{ }^{\circ} \mathrm{C}$. The resulting fragment was ligated with the pGEM-T Easy vector and used to transform E. coli $\mathrm{DH} 5 \alpha$ (Invitrogen). Mutants were selected on LB agar containing kanamycin $\left(50 \mu \mathrm{g} \mathrm{m} \mathrm{ml}^{-1}\right)$. Plasmid DNA was restriction-digested with Bam $\mathrm{HI}$ (NEB) and the fragment containing the $\mathrm{Kn}^{\mathrm{R}}$ cassette was purified using a Wizard SV gel and PCR cleanup kit (Promega).

The BamHI-digested fragment containing the $\mathrm{Kn}^{\mathrm{R}}$ cassette was ligated with the BclI-digested plasmid containing the $c d t$ genes, and used to transform E. coli DH5 $\alpha$. This construct was mobilized into C. jejuni using pGEM-T Easy vector (Promega) as it contains an E. coli origin of replication and cannot replicate in Campylobacter. Mutants that are kanamycin resistant arise as a result of a double homologous recombination event, in which the wild-type $c d t$ operon is replaced with the $c d t$ deletion containing the $\mathrm{Kn}^{\mathrm{R}}$ cassette. C. jejuni 81-176 was transformed by electroporation as previously described (Miller et al., 1988). Briefly, C. jejuni was grown in Columbia broth (Difco). Early-exponential-phase cells were rinsed twice with electroporation buffer (EPB: $272 \mathrm{mM}$ sucrose, $15 \%$, v/v, glycerol, $2.43 \mathrm{mM} \mathrm{K}_{2} \mathrm{HPO}_{4}$, $\left.0.57 \mathrm{mM} \mathrm{KH}_{2} \mathrm{PO}_{4}\right)$. C. jejuni was resuspended in $\mathrm{EPB}$ and incubated in a $0.1 \mathrm{~cm}$ cuvette for $10 \mathrm{~min}\left(4{ }^{\circ} \mathrm{C}\right)$ with plasmid DNA $(0.5 \mu \mathrm{g})$. A high-voltage pulse $\left(12.5 \mathrm{kV} \mathrm{cm}^{-1}\right)$ was delivered using an Electroporator 2510 (Brinkmann). Cells were immediately transferred to Karmali agar and allowed to recover for $4 \mathrm{~h}\left(37^{\circ} \mathrm{C}\right.$, microaerophilic atmosphere). Cells were then harvested and plated onto Karmali agar containing kanamycin $\left(30 \mu \mathrm{g} \mathrm{ml} \mathrm{m}^{-1}\right)$. Transformed colonies were screened by PCR analysis to confirm the deletion of $c d t B$ and insertion of the $\mathrm{Kn}^{\mathrm{R}}$ cassette (Purdy et al., 2000).

The phenotype of the CDT mutant was confirmed by inoculating T84 cells grown on chamber slides ( $70 \%$ confluent) with strain $81-176$ or the CDT-deletion mutant at a m.o.i. of 10 . Slides treated with sterile growth medium served as controls. After $30 \mathrm{~h}$ incubation, cells were fixed overnight with $2 \%(\mathrm{w} / \mathrm{v})$ paraformaldehyde and stained with Hoechst 33258 ( $1 \mu \mathrm{M}$, Molecular Probes) for $25 \mathrm{~min}$. Monolayers were rinsed once with phosphate-buffered saline (PBS). Slides were visualized by epifluorescent microscopy. Nuclei were enumerated and average nucleus area was determined by image analysis using Image Pro Plus v.4.1 software (Media Cybernetics). The patches of the monolayer where cells were obviously damaged and detached from the slide are referred to as 'death foci'.

Construction and characterization of the isogenic FlaAFlaB mutant. The presence of a functional flagellar apparatus has been previously shown to be required for maximal invasion of epithelial cells (Konkel et al., 2004). A non-invasive flagellar mutant of $C$. jejuni NCTC 11168 was constructed by deleting part of the flaA and flaB genes and inserting a $\mathrm{Kn}^{\mathrm{R}}$ cassette into the deletion site. The primers used to generate the mutant did not amplify the flaA and $f l a B$ genes from $C$. jejuni 81-176, necessitating the use of the sequenced strain NCTC 11168 . The mutant was constructed by amplifying the flaA and flaB genes from C. jejuni 11168 using primers flaAflaBF $\left(5^{\prime}\right.$ TGCTAAAGCAAACGCTGATT- $\left.3^{\prime}\right)$ and flaAflaBR $\left(5^{\prime}\right.$ CTGATTGCGCAAGGATGTTA-3'). PCR amplification was carried out as described above, except that the annealing temperature was $54{ }^{\circ} \mathrm{C}$ and the extension cycle was $3.5 \mathrm{~min}$. The resulting fragment was ligated with the pGEM-T Easy vector (Promega) and used to transform E. coli $\mathrm{DH} 5 \alpha$. Mutants were selected on LB agar containing ampicillin $\left(100 \mu \mathrm{g} \mathrm{ml}^{-1}\right)$. Plasmid DNA was extracted and digested with $E c o R V$ to generate the deletion.

A Kn ${ }^{\mathrm{R}}$ cassette was amplified, cloned and purified as described above except that the primers contained an EcoRV restriction site in place of the BamHI site. The EcoRV-digested $\mathrm{Kn}^{\mathrm{R}}$ cassette was ligated with the EcoRV-digested plasmid containing the flaA flaB genes, and used to transform E. coli DH5 $\alpha$. This construct was mobilized into C. jejuni NCTC 11168 as described above. Transformed colonies were screened by PCR analysis to confirm the partial deletion of flaA flaB and insertion of the $\mathrm{Kn}^{\mathrm{R}}$ cassette. Motility was also examined by phasecontrast microscopy and by assessing swarming on motility agar (Columbia agar containing $0.4 \%$ agar). The low percentage of agar allows motile bacteria to move within the agar forming a halo of growth.

Inoculation protocol. Confluent monolayers were rinsed twice with pre-warmed $\left(37^{\circ} \mathrm{C}\right)$ Dulbecco's phosphate buffered saline (PBS) (Sigma) and antibiotic-free, phenol-red-free culture medium was added to each well. Monolayers were inoculated with C. jejuni (cultured as described above) to achieve a m.o.i. of 100 c.f.u. per enterocyte. Control monolayers received an equivalent volume of sterile growth medium. The topoisomerase-I inhibitor camptothecin ( $4 \mu \mathrm{g} \mathrm{ml}^{-1}$ in DMSO vehicle, Sigma) and the oxidant $\mathrm{H}_{2} \mathrm{O}_{2}(0.5 \mathrm{mM}$, Sigma) were used as induction control treatments for apoptosis and oncosis, respectively (Kiechle \& Zhang, 2002; Palomba et al., 1999). To determine the possible involvement of caspases in C. jejuniinduced cell death, T84 monolayers were pre-treated with the pancaspase inhibitor Z-VAD-FMK $(120 \mu \mathrm{M}$ in DMSO vehicle, Calbiochem) for $1 \mathrm{~h}\left(37^{\circ} \mathrm{C}, 5 \% \mathrm{CO}_{2}\right)$ prior to inoculation with $C$. jejuni, and inhibitors remained present throughout the $C$. jejuni challenge as described previously (Chin et al., 2003).

Oligonucleosomal DNA fragmentation assay. Oligonucleosomal DNA fragmentation was quantified using a Cell Death Detection ELISA kit (Roche Molecular Biochemicals). This immunoassay detects histone-DNA complexed fragments (oligonucleosomes) present in the cytoplasm of apoptotic cells. Resultant absorbance values are proportional to the amount of oligonucleosomes present in the cytoplasm of cell lysates. T84 monolayers were grown in 48-well plates and inoculated with $C$. jejuni or sterile broth as described above. After challenge, medium was aspirated and oligonucleosomal DNA fragmentation in cell lysates was determined according to the manufacturer's protocol. DNA fragmentation is expressed as $A_{405}$ units. According to the manufacturer, the detection limit for this ELISA is $10^{2}$ apoptotic cells.

Cytotoxicity assay. The release of the cytoplasmic enzyme lactate dehydrogenase (LDH) into the surrounding culture medium is an indicator of plasma membrane disruption (Brennan \& Cookson, 2000). T84 monolayers were grown in 48 -well plates and inoculated with C. jejuni or sterile broth as described above. Culture medium was analysed for the presence of LDH using a Cytotoxicity Detection kit (Roche Molecular Biochemicals) according to the manufacturer's protocol. Total and spontaneously released LDH activity was also determined. Results are presented as percentage cytotoxcity, which 
was calculated as [(experimental LDH-spontaneously released LDH)/ (total LDH-spontaneously released $\mathrm{LDH})] \times 100$.

Measurement of intracellular ATP. T84 monolayers were grown in 48-well plates and inoculated as described above. Intracellular ATP levels were determined using an ATP Bioluminescence Assay kit CLS II (Roche Molecular Biochemicals) according to the manufacturer's protocol. Luminescence (10 s integration) was measured using a Lumat LB 5907 luminometer (EG\&G Berthold). A standard curve generated from known concentrations of ATP was used to calculate the ATP content of each sample. The cellular ATP content is expressed relative to the cell number.

Mitochondrial transmembrane potential. To visually assess the mitochondrial transmembrane potential of treated T84 monolayers, a JC-1 mitochondrial membrane potential detection kit (Cell Technology) was used according to the manufacturer's protocol. JC-1 is a cell permeant dye that aggregates within mitochondria of healthy cells and forms a red-fluorescent multimeric complex (Smiley et al., 1991). Conversely, in cells that have a low mitochondrial transmembrane potential, the dye is dispersed within the cytoplasm and remains in its fluorescent green monomeric form. Briefly, T84 cells were grown to $90 \%$ confluence on chamber slides and treated with $C$. jejuni as described above, with camptothecin $\left(4 \mu \mathrm{g} \mathrm{ml}^{-1}\right)$, or $\mathrm{H}_{2} \mathrm{O}_{2}(0.5 \mathrm{mM})$. After incubation $(4 \mathrm{~h})$, monolayers were rinsed three times with PBS, and then DMEM containing JC-1 reagent and Hoechst 33258 ( $1 \mu \mathrm{M}$, Molecular Probes; $100 \mu \mathrm{l}$ per well) was added to each well and incubated at $37{ }^{\circ} \mathrm{C}, 5 \% \mathrm{CO}_{2}$ for $25 \mathrm{~min}$. Monolayers were rinsed once with JC-1 assay buffer. Slides were visualized by epifluorescent microscopy.

Western blot analysis. T84 monolayers were grown in six-well plates and treated with $C$. jejuni as described above or with camptothecin $\left(4 \mu \mathrm{g} \mathrm{ml}^{-1}\right)$. After incubation, monolayers were rinsed twice with PBS containing $1 \mathrm{mM}$ EGTA (Sigma) and protease inhibitor cocktail $\left(25 \mu \mathrm{l} \mathrm{ml}^{-1} ; \mathrm{P}\right.$ 8340, Sigma). Proteins were extracted from the monolayers by boiling the samples for $10 \mathrm{~min}$ in sample buffer $(0.125 \mathrm{M}$ Tris/HCl pH 6.8, $4 \%$ SDS, $20 \%$, v/v, glycerol, 1.44 M 2-mercaptoethanol; Sigma), and stored at $-80{ }^{\circ} \mathrm{C}$ until use. Proteins were separated by $10 \%$ SDS-PAGE and transferred onto a $0.45 \mu \mathrm{M}$ nitrocellulose membrane (Bio-Rad). Membranes were blocked for $1 \mathrm{~h}$ in TBS containing $5 \%$ skim milk and $0.1 \%$ Tween 20 and then rinsed briefly in TBS containing Tween 20 (TTBS). Membranes were incubated for $1 \mathrm{~h}$ at room temperature in TTBS containing $2 \%$ BSA, and mouse anti-PARP antibody $(1 / 1500$ dilution, Roche) or mouse anti- $\alpha$-tubulin antibody (1/3000 dilution, Molecular Probes) as the internal control. Membranes were washed with TTBS and then incubated for $1 \mathrm{~h}$ at room temperature in TTBS containing $2 \% \mathrm{BSA}$, and horseradish-peroxidase-conjugated antimouse IgG antibody (1:5000 dilution, Sigma). Membranes were washed with TTBS and visualized by ECL detection (Amersham Pharmacia). Band densities were determined by image analysis using AlphaEaseFC software, v3.2.1 (Alpha Inotech).

Invasion and adherence assays. T84 monolayers were grown in 48-well plates and inoculated as described above. After incubation, infected monolayers were rinsed three times with PBS. To assess adherence, monolayers were lysed with $0.1 \%$ Triton X-100 in PBS for $10 \mathrm{~min}$ at room temperature on an orbital shaker. Following lysis, bacteria were enumerated by plating tenfold serial dilutions onto Karmali agar. Invasion was determined using a gentamicin protection assay. After incubation, infected monolayers were rinsed three times with PBS. Monolayers were then incubated for $3 \mathrm{~h}$ with fresh tissue culture medium containing gentamicin $\left(500 \mu \mathrm{g} \mathrm{ml} \mathrm{m}^{-1}\right)$ to kill extracellular bacteria. Following incubation, monolayers were rinsed, lysed, and bacteria were enumerated as for the adherence assay. A preliminary experiment was conducted to ensure that a bactericidal concentration of gentamicin was used for the invasion assay. C. jejuni $81-176\left(7.5 \times 10^{7}\right.$ c.f.u.) treated for $3 \mathrm{~h}$ with 100,250 and $500 \mu \mathrm{g}$ gentamicin $\mathrm{ml}^{-1}$ resulted in the recovery of 960,276 and 22 c.f.u., confirming previously reported values (Monteville et al., 2003). Subsequent invasion assays were conducted with $500 \mu \mathrm{g}$ gentamicin $\mathrm{ml}^{-1}$.

Statistical analysis. All statistical calculations were performed with GraphPad InStat v.3.06 software (GraphPad Software). For each experiment, repetitions were conduced on separate occasions. Data are expressed as the means \pm SEM, and compared by one-way analysis of variance, followed by the Tukey-Kramer multiple comparison test. Regression analysis was performed using Pearson correlation analysis. Statistical significance was established at a $P$ value of $<0.05$.

\section{RESULTS}

\section{C. jejuni-induced enterocyte death is consistent with oncosis}

The effect of C. jejuni on enterocyte morphology was initially examined by transmission electron microscopy. Six hours post-inoculation, C. jejuni-treated T84 enterocytes appeared degenerated and exhibited extensive cytoplasmic vacuolation (results not shown). A loss of interdigitation between adjacent cells, and detached cells, were often observed. Nuclei were barely distinguishable from the cytoplasm, and nuclear condensation or fragmentation was not evident. Remarkably, cells exhibiting classic apoptotic morphology (i.e. condensed and fragmented nuclei) were conspicuously absent in C. jejuni-treated monolayers (100 cells from each of five separate regions of the monolayer were observed per treatment). In contrast, control monolayers exhibited well-defined nuclei and interdigitation between adjoining cells. Occasionally, as expected, cells exhibiting classic apoptotic morphology were observed in the control-treated monolayers ( $\sim \%$ of cells observed).

C. jejuni induced a time-dependent increase in oligonucleosomal DNA fragmentation that was significantly higher compared to that seen with the control treatment from $6 \mathrm{~h}$ onwards (Fig. 1a). Monolayers treated with pro-apoptotic camptothecin also exhibited significant DNA fragmentation from $3 \mathrm{~h}$ onwards. Significant DNA fragmentation was exhibited in monolayers treated with pro-oncotic $\mathrm{H}_{2} \mathrm{O}_{2}$ at 3 and $6 \mathrm{~h}$ post-treatment. Oligonucleosomes were not detected by ELISA in lysates of $C$. jejuni in the absence of enterocytes, thus confirming that this assay was specific to epithelial cell DNA fragmentation (results not shown).

C. jejuni-treated monolayers exhibited significantly increased cytotoxicity from $3 \mathrm{~h}$ post-inoculation onwards, compared to the control treatment (Fig. 1b). Monolayers treated with $\mathrm{H}_{2} \mathrm{O}_{2}$, an inducer of oncosis (Palomba et al., 1999), exhibited significant cytotoxicity at $1.5 \mathrm{~h}$ (earliest time point measured). Cytotoxicity was not significantly different between the control and pro-apoptotic camptothecin treatments. In a preliminary study (in the absence of enterocytes) it was determined that $C$. jejuni does not 

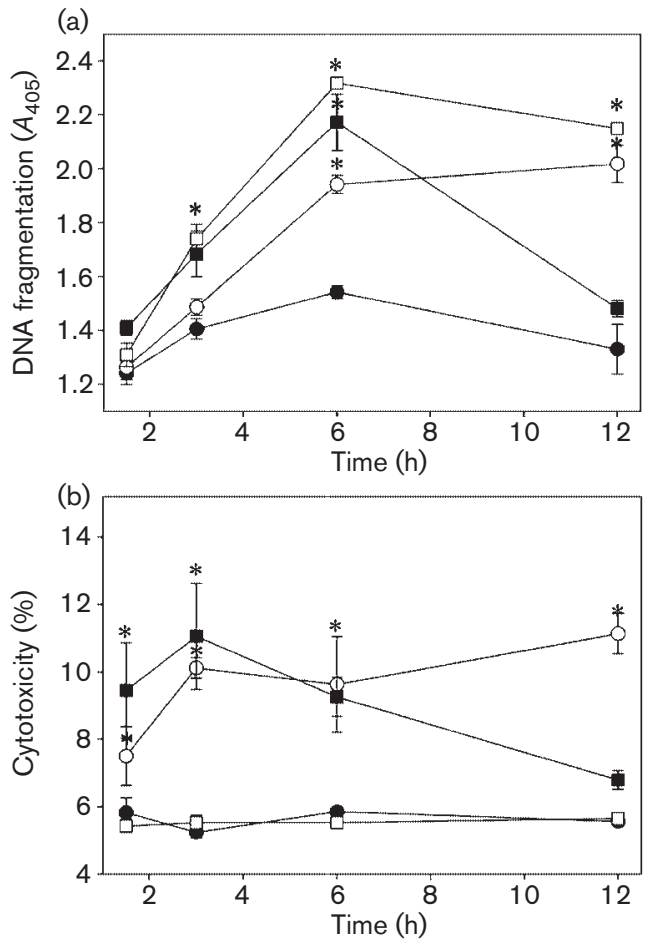

Fig. 1. C. jejuni 81-176 induces rapid enterocyte cytotoxicity and oligonucleosomal DNA fragmentation. T84 monolayers were incubated with sterile growth medium (control, ๑), C. jejuni $(\bigcirc)$, $\mathrm{H}_{2} \mathrm{O}_{2}$ (ם) or camptothecin ( $\square$ ). Oligonucleosomal DNA fragmentation (a) and cytotoxicity (b) were determined at various times. Values are means \pm SEM for three replicates (six observations per treatment). ${ }^{*}, P<0.05$ vs control treatment.

possess detectable LDH activity of its own (results not shown).

As cell death is largely regulated by mitochondria, experiments were conducted to determine the effect of $C$. jejuni on mitochondrial transmembrane potential. Aggregation of red-fluorescent JC-1 dye was observed in the mitochondria of the control-treated monolayers (Fig. 2a). In contrast, C. jejuni-, $\mathrm{H}_{2} \mathrm{O}_{2^{-}}$, and camptothecin-treated monolayers exhibited a diffuse green fluorescence, indicating the collapse of mitochondrial transmembrane potential. Green JC-1 staining was more intense in C. jejuni- and $\mathrm{H}_{2} \mathrm{O}_{2}$-treated cells, consistent with increased dye uptake as a result of the loss of plasma membrane integrity in these cells. As mitochondria serve as the major site for the generation of ATP, intracellular ATP was also measured in treated T84 monolayers. ATP levels were significantly reduced in $C$. jejuni-infected monolayers (6 h) compared to the control treatment (Fig. 2b). Significantly decreased intracellular ATP levels were also observed in mononlayers treated with either $\mathrm{H}_{2} \mathrm{O}_{2}$ or campothecin compared to controls.

Since caspases mediate many aspects of cell death and inhibition of caspase-1 has been shown to prevent the
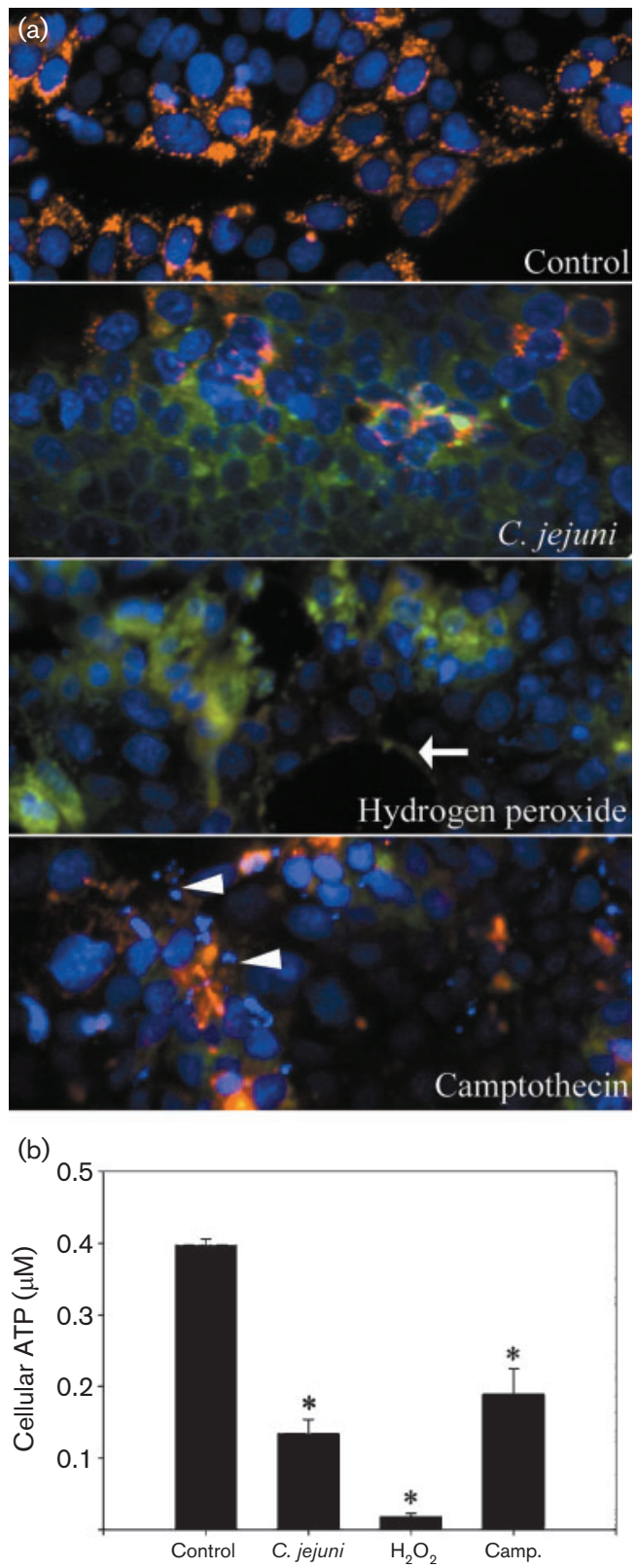

Fig. 2. C. jejuni 81-176 causes mitochondrial transmembrane depolarization and depletion of intracellular ATP in enterocytes. (a) Representative images of mitochondrial transmembrane potential in T84 cells. T84 monolayers were treated for $6 \mathrm{~h}$ with sterile growth medium, C. jejuni, $\mathrm{H}_{2} \mathrm{O}_{2}$ or camptothecin and stained with $\mathrm{JC}-1$. The red-fluorescent aggregated form of JC-1 indicates the presence of a high mitochondrial transmembrane potential, whereas cytoplasmic staining with the green fluorescent monomeric form of JC-1 indicates the loss of mitochondrial transmembrane potential. The arrow indicates where cells have sloughed off from the monolayer. Arrowheads indicate nuclear chromatin condensation and fragmentation. The figure shows representative images from one of two replicates. (b) Intracellular ATP levels of treated T84 monolayers were determined $6 \mathrm{~h}$ postinoculation. Values are means \pm SEM for three replicates (three observations per treatment). ${ }^{*}, P<0.05$ vs control treatment. 
Salmonella-induced oncosis (or 'pyroptosis') of macrophages (Brennan \& Cookson, 2000), the involvement of caspases in C. jejuni-induced death was examined. Treatment of $C$. jejuni-infected monolayers with the pancaspase inhibitor Z-VAD-FMK did not prevent oligonucleosomal DNA fragmentation (Fig. 3a). In contrast, caspase inhibition prevented the DNA fragmentation induced by both pro-apoptotic camptothecin and prooncotic $\mathrm{H}_{2} \mathrm{O}_{2}$. Pan-caspase inhibition did not prevent $C$. jejuni-induced cytotoxicity (Fig. 3b). Caspases also mediate the cleavage of PARP in apoptotic cells (Los et al., 2002). In C. jejuni-treated T84 cells, PARP was present in its uncleaved state $(116 \mathrm{kDa})$ and the PARP-cleavage fragment $(89 \mathrm{kDa})$ was not detected throughout infection even up to $36 \mathrm{~h}$ post-inoculation (Fig. 3c). In contrast, cleaved PARP was apparent in the camptothecin-treated monolayers, in which a time-dependent loss of the $116 \mathrm{kDa}$ fragment was observed. Cleaved vs intact PARP ratios were significantly greater in camptothecin-treated cells ( $36 \mathrm{~h}$ ) compared to values of monolayers exposed to $C$. jejuni for 36 h (Fig. 3d).

\section{C. jejuni induces enterocyte death independent of CDT expression}

The CDT deletion mutant was characterized to ensure that characteristic cytotoxic effects of the CDT were absent. PCR analysis of the CDT deletion mutant confirmed the absence of the $c d t B$ gene and insertion of the $\mathrm{Kn}^{\mathrm{R}}$ cassette (results not shown). PCR analysis also demonstrated the absence of plasmid DNA (used for construction of the mutant), confirming that the mutant was the product of a double homologous recombination event. After $30 \mathrm{~h}$, T84 cells treated with the wild-type 81-176 displayed effects consistent with those previously described for CDTintoxicated cells (Purdy et al., 2000; Whitehouse et al., 1998). Namely, cells were greatly distended (Fig. 4a), there was a significant reduction in the number of cells undergoing mitosis, and cells displayed nuclear distension consistent with G2/M-phase cell cycle arrest (Table 1). Compared to the control treatment, there were significantly fewer cells per field of view corresponding to a combination of cell cycle arrest and increased oncosis. In contrast, T84 cells inoculated with the CDT mutant were (a)
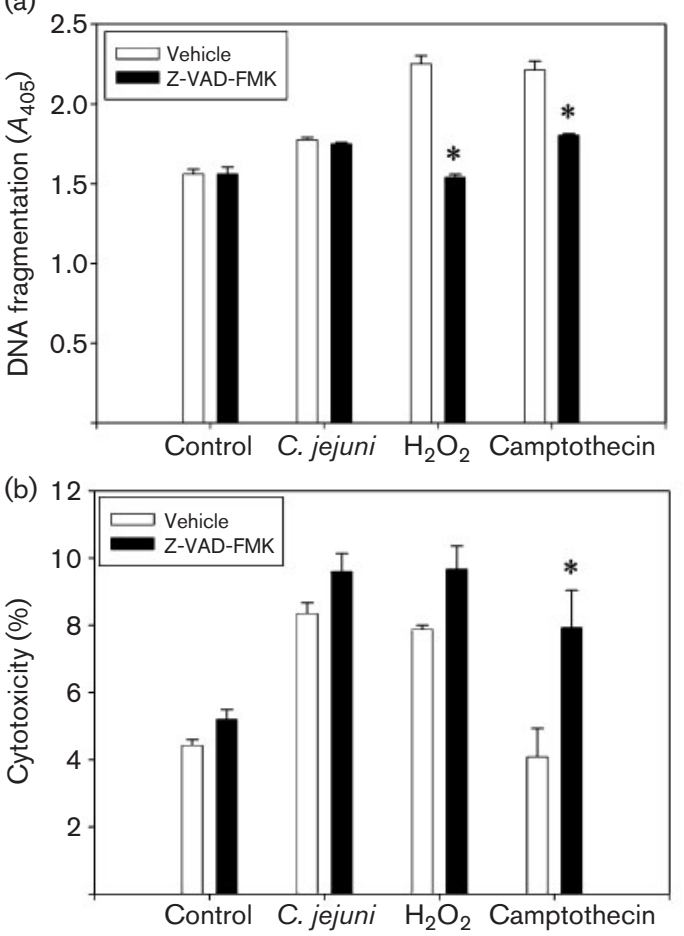

(c)

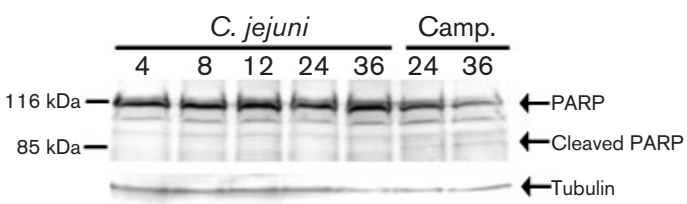

(d)

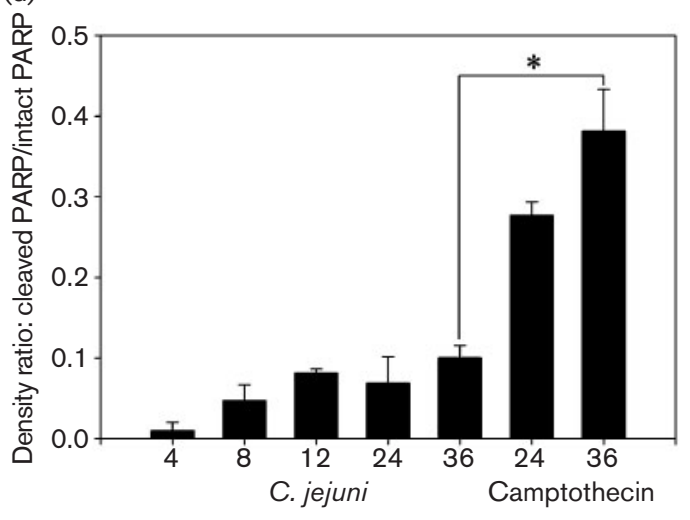

Fig. 3. $(a, b) C$. jejuni-induced enterocyte death is caspase independent. T84 monolayers were inoculated with sterile growth medium, C. jejuni 81-176, $\mathrm{H}_{2} \mathrm{O}_{2}$ or camptothecin in the presence or absence of a pan-caspase inhibitor, Z-VAD-FMK. Oligonucleosomal DNA fragmentation (a) and cytotoxicity (b) were determined $6 \mathrm{~h}$ post-inoculation. Values are means \pm SEM for three replicates (six observations per treatment). ${ }^{*}, P<0.05$ vs vehicle treatment. (c) PARP remains in its active, non-cleaved form in C. jejuni-treated T84 monolayers. T84 monolayers were incubated with C. jejuni 81-176 or pro-apoptotic camptothecin (control treatment) for various times and PARP was detected by Western blotting. Results were consistent across replicates and a representative blot from one of three replicates is shown. Western blots were quantified by densitometry (d); values are means \pm SEM for three replicates. ${ }^{*}, P<0.05$ vs control treatment $(36 \mathrm{~h})$. 
(a)
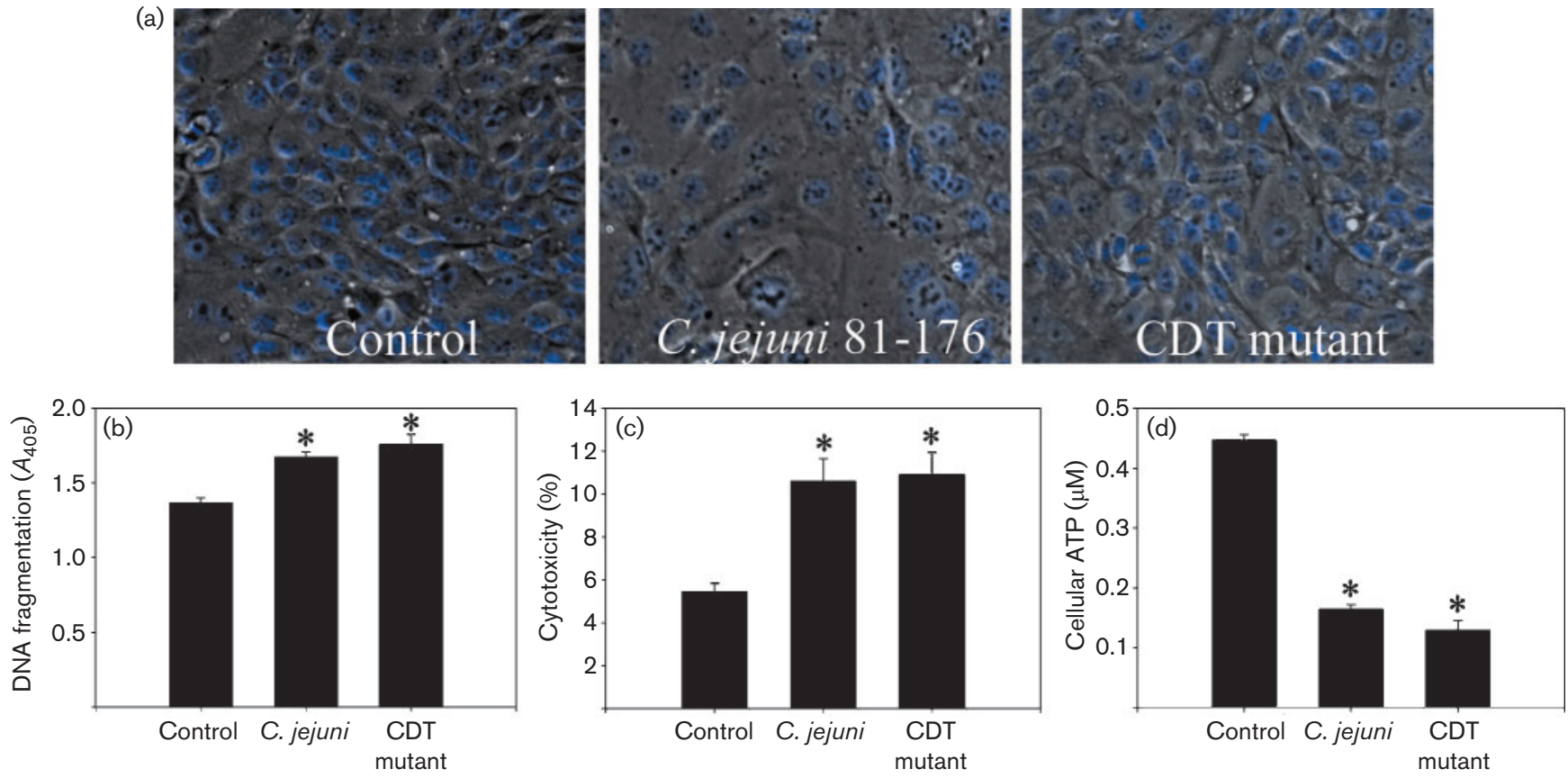

Fig. 4. C. jejuni induces enterocyte death independent of CDT expression. (a) Characteristic distension of intestinal epithelial cells was observed in T84 cells treated with $C$. jejuni 81-176 but not with the isogenic CDT deletion mutant. Epithelial cells treated with sterile growth medium served as a negative control. A representative image (differential interference contrast) is shown from one of three replicates (three observations per treatment). Nuclei are stained blue. (b-d) T84 monolayers were incubated with sterile growth medium, C. jejuni or the CDT mutant and assayed for oligonucleosomal DNA fragmentation (b), cytotoxicity (c), or cellular ATP concentration (d). Values are means \pm SEM for three replicates (three to six observations per treatment). ${ }^{*}, P<0.05$ vs control treatment.

not characteristically distended (Fig. 4a) and the number of mitotic cells did not differ from the control treatment (Table 1). There were significantly fewer cells per field of view than for the control treatment, but significantly more cells for the wild-type $C$. jejuni treatment (i.e. corresponding

Table 1. Comparison of cytotoxic effects of wild-type C. jejuni 81-176 and the isogenic CDT mutant

Semi-confluent T84 cells were grown on chamber slides and inoculated with C. jejuni 81-176 and the CDT mutant at a m.o.i. of 10 and incubated for $30 \mathrm{~h}$. T84 cells inoculated with sterile growth medium served as a negative control. Microscopy and image analysis were performed as outlined in Methods. Results are means \pm SEM for three replicates (three observations per treatment).

\begin{tabular}{|lcccc|}
\hline & $\begin{array}{c}\text { T84 cells } \\
\text { per field } \\
\text { of view }\end{array}$ & $\begin{array}{c}\text { Average area } \\
\text { of nucleus } \\
\left(\boldsymbol{\mu \mathbf { m } ^ { 2 } )}\right.\end{array}$ & $\begin{array}{c}\text { Mitotic } \\
\text { cells per } \\
\text { field of view }\end{array}$ & $\begin{array}{c}\text { Death foci } \\
\text { per field } \\
\text { of view }\end{array}$ \\
\hline Neg. control & $1144 \pm 40$ & $3.92 \pm 0.23$ & $12.7 \pm 3.2$ & $0 \pm 0$ \\
$81-176$ & $660 \pm 49^{\star}$ & $4.69 \pm 0.22$ & $0.7 \pm 0.3^{\star}$ & $5.3 \pm 1.5^{\star}$ \\
CDT mutant & $865 \pm 75^{\star} \dagger$ & $4.04 \pm 0.28$ & $7.0 \pm 1.0 \dagger$ & $5.6 \pm 1.5^{\star}$ \\
\hline
\end{tabular}

${ }^{\star} P<0.05$ vs negative control treatment.

$\dagger P<0.05$ vs C. jejuni 81-176. to oncosis but not oncosis plus cell cycle arrest). Patches of the monolayer containing damaged or detached cells ('death foci') were also observed in both C. jejuni- and CDT mutant-treated monolayers.

The role of CDT in enterocyte oncosis was examined by comparing DNA fragmentation, cytotoxicity and ATP levels in T84 monolayers exposed to wild-type C. jejuni or the CDT mutant. In monolayers co-incubated for $6 \mathrm{~h}$ with the CDT mutant, both oligonucleosomal DNA fragmentation and cytotoxicity were significantly increased (Fig. 4b, c) and cellular ATP was significantly reduced (Fig. 4d) compared to the control treatment. Values did not differ significantly between the CDT mutant and wildtype C. jejuni. Deletion of the CDT gene from strain 81-176 also had no effect on T84 invasion $(1.122 \pm 0.165$ vs $1.143 \pm 0.158 \%$ of the initial inoculum was internalized in T84 monolayers after $2 \mathrm{~h}$ of co-incubation with strain 81 176 and the CDT mutant respectively).

\section{C. jejuni-induced enterocyte oncosis is strain specific and is correlated with greater levels of host cell invasion}

To determine whether other strains of $C$. jejuni are capable of inducing oncosis, and investigate the relationship 
between cytotoxicity and cell invasion, T84 monolayers were inoculated with 23 clinical isolates of $C$. jejuni, and the pro-oncotic release of cytosolic LDH into the surrounding culture medium ('cytotoxicity') and the corresponding invasion of the cells were measured from the same monolayer. Five clinical isolates (CHR 31, 34, 58, 87, and 107) and strain 81-176 demonstrated significant cytotoxic LDH release from T84 cells within $3 \mathrm{~h}$ (Table 2). Cytotoxicity was significantly correlated with greater levels of host cell invasion $\left(y=2.36 x+6.56, r^{2}=0.743, P<0.0001\right.$; Fig. 5). The $c d t B$ gene was present in all clinical isolates except one (CHR 263) as determined by PCR analysis (not shown).

To further address whether invasion plays a role in oncotic death, C. jejuni NCTC 11168 and an isogenic flagellar

Table 2. C. jejuni-induced cytotoxicity and invasion of T84 monolayers

Confluent T84 monolayers were inoculated with strain $81-176$ or one of 23 clinical C. jejuni isolates obtained from patients with enteritis (Chinook Health Region). Cytotoxicity and invasion were determined $3 \mathrm{~h}$ post-inoculation using the same monolayer. Values are the mean \pm SEM of five replicates for each isolate (six observations). Only half of the isolates were assayed at one time (each time contained a control treatment and strain 81-176 (=reference). Values for the control and 81-176 did not differ between times.

\begin{tabular}{|c|c|c|c|}
\hline Treatment & $\begin{array}{c}\text { Cytotoxicity } \\
(\%)\end{array}$ & $\begin{array}{c}\text { Control } \\
\text { cytotoxicity } \\
(\%)\end{array}$ & $\begin{array}{c}\text { Internalized } C \text {. } \\
\text { jejuni (\% of } \\
\text { initial inoculum) }\end{array}$ \\
\hline CHR 10 & $7.04 \pm 0.99$ & $5.37 \pm 0.30$ & $0.25 \pm 0.11^{\star}$ \\
\hline CHR 28 & $7.31 \pm 0.41$ & $5.21 \pm 0.71$ & $0.48 \pm 0.17^{\star}$ \\
\hline CHR 30 & $8.31 \pm 0.54$ & $5.37 \pm 0.30$ & $0.25 \pm 0.03^{\star}$ \\
\hline CHR 31 & $9.58 \pm 0.98 \dagger$ & $5.37 \pm 0.30$ & $0.64 \pm 0.14^{\star}$ \\
\hline CHR 32 & $6.96 \pm 0.57$ & $5.37 \pm 0.30$ & $0.13 \pm 0.06^{*}$ \\
\hline CHR 33 & $6.75 \pm 0.45$ & $5.21 \pm 0.71$ & $0.52 \pm 0.08^{\star}$ \\
\hline CHR 34 & $9.40 \pm 0.91 \dagger$ & $5.37 \pm 0.30$ & $1.54 \pm 0.52$ \\
\hline CHR 36 & $7.91 \pm 0.35$ & $5.21 \pm 0.71$ & $0.52 \pm 0.10^{*}$ \\
\hline CHR 58 & $10.81 \pm 0.88 \dagger$ & $5.21 \pm 0.71$ & $1.70 \pm 0.23$ \\
\hline CHR 87 & $9.51 \pm 1.41 \dagger$ & $5.37 \pm 0.30$ & $1.05 \pm 0.27$ \\
\hline CHR 94 & $6.92 \pm 1.07$ & $5.21 \pm 0.71$ & $0.39 \pm 0.16^{*}$ \\
\hline CHR 107 & $9.93 \pm 0.97 \dagger$ & $5.21 \pm 0.71$ & $1.55 \pm 0.08$ \\
\hline CHR 113 & $6.48 \pm 0.36$ & $5.21 \pm 0.71$ & $0.43 \pm 0.18^{\star}$ \\
\hline CHR 158 & $7.84 \pm 0.72$ & $5.37 \pm 0.30$ & $0.16 \pm 0.04^{*}$ \\
\hline CHR 189 & $6.91 \pm 0.25$ & $5.21 \pm 0.71$ & $0.50 \pm 0.03^{\star}$ \\
\hline CHR 213 & $6.50 \pm 0.69$ & $5.37 \pm 0.30$ & $0.17 \pm 0.05^{\star}$ \\
\hline CHR 216 & $7.19 \pm 0.65$ & $5.21 \pm 0.71$ & $0.48 \pm 0.09^{\star}$ \\
\hline CHR 218 & $6.71 \pm 0.20$ & $5.21 \pm 0.71$ & $0.20 \pm 0.08^{\star}$ \\
\hline CHR 225 & $6.95 \pm 0.38$ & $5.21 \pm 0.71$ & $0.26 \pm 0.08^{*}$ \\
\hline CHR 234 & $7.41 \pm 0.20$ & $5.37 \pm 0.30$ & $0.17 \pm 0.02^{\star}$ \\
\hline CHR 245 & $6.88 \pm 0.59$ & $5.37 \pm 0.30$ & $0.24 \pm 0.07^{\star}$ \\
\hline CHR 263 & $8.07 \pm 0.38$ & $5.37 \pm 0.30$ & $0.24 \pm 0.09^{*}$ \\
\hline CHR 277 & $7.92 \pm 0.58$ & $5.37 \pm 0.30$ & $0.53 \pm 0.09^{*}$ \\
\hline $81-176$ & $11.09 \pm 0.70 \dagger$ & $5.47 \pm 0.51$ & $1.59 \pm 0.07$ \\
\hline
\end{tabular}

${ }^{*} P<0.05$ vs strain $81-176$.

$\dagger P<0.05$ vs control treatment.

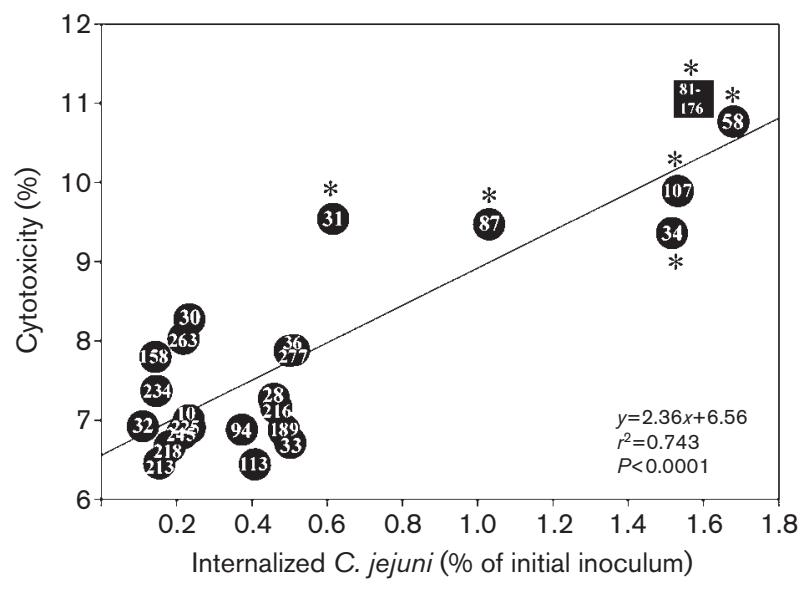

Fig. 5. C. jejuni-induced cytotoxicity is correlated with higher levels of invasiveness. T84 monolayers were inoculated with strain 81-176 or one of 23 clinical C. jejuni isolates obtained from patients with enteritis (Chinook Health Region). Cytotoxicity and invasion were determined $3 \mathrm{~h}$ post-inoculation using the same monolayer. Each point represents the mean value of five replicates for each isolate (six observations). ${ }^{*}, P<0.05$ vs the negative control treatment for cytotoxicity.

mutant were assessed for their ability to adhere and invade T84 enterocytes and cause cytotoxicity. The primers used to generate the mutant did not amplify the flaA and $f l a B$ genes from strain 81-176, necessitating the use of strain NCTC 11168 for generating a non-invasive mutant. The FlaAFlaB mutant was non-motile as determined by phasecontrast microscopy and swarming on motility agar (Fig. 6a). Cytotoxicity, adhesion and invasion were determined $3 \mathrm{~h}$ post-inoculation using the same monolayer. Cytotoxicity was significantly increased in T84 monolayers treated with the wild-type strain NCTC 11168 compared to control- and FlaAFlaB mutant-treated monolayers (Fig. 6b). Also, cytoxicity did not differ significantly between control- and FlaAFlaB mutanttreated monolayers. Compared to the wild-type strain, the FlaAFlaB mutant exhibited significantly decreased ability to adhere to T84 cells $(11.20 \pm 0.61$ vs $0.62 \pm 0.042 \%$ of the initial inoculum adhered to T84 monolayers respectively). The FlaAFlaB mutant also exhibited significantly decreased ability to invade T84 cells $(0.55 \pm 0.12$ vs $0.0044 \pm 0.0010 \%$ of the initial inoculum of the wild-type and FlaAFlaB mutant invaded T84 monolayers respectively). The $c d t B$ gene was present in strain NCTC 11168 as determined by PCR.

\section{DISCUSSION}

Enteric pathogens have evolved numerous strategies to circumvent the intestinal epithelial barrier and colonize their host. While several studies have described cytotoxic effects of C. jejuni, particularly the effects of its CDT, on 

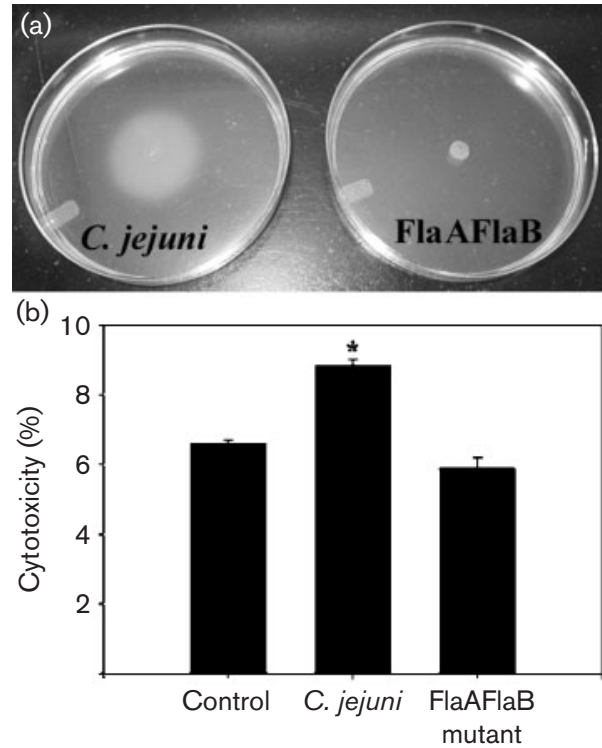

Fig. 6. A non-adherent and non-invasive FlaAFlaB mutant of $C$. jejuni NCTC 11168 is defective in its ability to cause T84 enteroctyte oncosis. (a) The flagellar FlaAFlaB mutant is non-motile in soft agar (24 h). (b) Cytotoxicity is significantly increased in T84 monolayers treated with the wild-type strain NCTC 11168 compared to control- and FlaAFlaB mutant-treated monolayers ( $3 \mathrm{~h}$ post-inoculation). Each point represents the mean value of three replicates for each isolate (six observations). ${ }^{*}, P<0.05$ vs the negative control treatment for cytotoxicity.

cultured epithelial cells, the mode by which C. jejuni may cause enterocyte death has not been adequately characterized. Results of this study indicate, apparently for the first time, that $C$. jejuni induces enterocyte oncosis in a straindependent manner, and that death is correlated with increased invasiveness and does not require the presence of the CDT.

Previous studies demonstrated that $C$. jejuni induced apoptosis of monocytes and lymphocytes (Hickey et al., 2005; Siegesmund et al., 2004; Zhu et al., 1999). In these studies, death occurred in a slow manner $(>24 \mathrm{~h})$ consistent with apoptosis. In contrast, we observed that C. jejuni induced rapid enterocyte death (3-6 h), inconsistent with apoptosis. Using a number of morphological and biochemical features to characterize the mode of cell death, we revealed that $C$. jejuni-treated enterocytes displayed features consistent with oncosis. C. jejuni-treated enterocytes exhibited cytoplasmic vacuolation, and nuclear condensation typical of apoptotic cells was not evident. Importantly, C. jejuni induced a rapid loss of plasma membrane integrity, which is a hallmark of oncosis. A similar rapid loss of plasma membrane integrity was previously noted in C. jejuni-treated HeLa epithelial cells (Newell \& Pearson, 1984). Oncotic disruption of the epithelial plasma membrane would release cellular contents into the internal milieu, consistent with the acute inflammatory response observed in many cases of campylobacteriosis. However, further investigation is needed to determine the degree to which C. jejuni-induced enterocyte oncosis is involved in inflammatory diarrhoea.

Caspases mediate many aspects of apoptosis, including DNA fragmentation (Earnshaw et al., 1999). While the morphological characteristics of C. jejuni-treated enterocytes were consistent with an oncotic death process, the cells also exhibited increased oligonucleosomal DNA fragmentation, a feature more typical of apoptosis. However, we also observed that C. jejuni-induced DNA fragmentation was independent of caspase activity, indicative of a non-apoptotic mode of death. Caspaseindependent DNA fragmentation has been previously observed in epithelial cells treated with a mitochondrial uncoupler that induces oncosis (Dong et al., 1997). In contrast, $\mathrm{H}_{2} \mathrm{O}_{2}$ induced caspase-dependent DNA cleavage. Although $\mathrm{H}_{2} \mathrm{O}_{2}$ is commonly used as an inducer of oncosis, it actually simultaneously causes both oncosis and apoptosis to varying degrees depending on the dose and extent of damage inflicted (Palomba et al., 1999). Thus, it is not unexpected that $\mathrm{H}_{2} \mathrm{O}_{2}$ induced significant caspasedependent DNA fragmentation in addition to its prooncotic effects. In camptothecin-treated cells, caspase inhibition prevented apoptosis (i.e. DNA fragmentation) as expected, whereas it potentiated oncosis (i.e. cytotoxicity), suggesting that death was not prevented but rather the mode of death was switched from apoptosis to oncosis. This is consistent with the notion that caspase inhibitors may promote alternative cell death pathways (i.e. oncosis or autophagy) (Vandenabeele et al., 2006), and that oncosis functions as the default death pathway when other forms of cell death are blocked (Golstein \& Kroemer, 2007). Indeed, previous studies have also observed that caspase inhibition induced cells to undergo oncosis despite treatment with an otherwise pro-apoptotic stimulus (Los et al., 2002; Vandenabeele et al., 2006).

Although caspases are primarily effectors of apoptotic death, caspase-1 has been shown to mediate Salmonellainduced macrophage oncosis (Brennan \& Cookson, 2000). However, C. jejuni-induced enterocyte oncosis was not prevented by caspase inhibition, suggesting that oncosis occurs via a caspase-independent mechanism that is distinct from that of other pathogens. Notably, C. jejuni also induces caspase-1 and -9-independent apoptosis of macrophages (Siegesmund et al., 2004).

We observed that $C$. jejuni caused disruption of the mitochondrial transmembrane potential and ATP depletion in association with its direct cytotoxic effects on enterocytes. Damaged mitochondria have been previously observed in cell culture and animal models of campylobacteriosis (De Melo et al., 1989; Gao et al., 1991; Humphrey et al., 1986; Newell \& Pearson, 1984), suggesting that $C$. jejuni may target mitochondria to induce enterocyte death. The targeting of mitochondria appears to be a common strategy used by many pathogens, including 
the closely related gastric pathogen Helicobacter pylori (Blanke, 2005). Mitochondria serve as executioners of both apoptotic and oncotic death (Kim et al., 2003). In this study, treatment of enterocytes with camptothecin, $\mathrm{H}_{2} \mathrm{O}_{2}$ or C. jejuni caused mitochondrial transmembrane depolarization and ATP depletion. However, apoptotic features such as maintenance of plasma membrane integrity and PARP cleavage were only observed in camptothecin-treated cells. These results are consistent with the known mechanisms of action for these agents. Camptothecin causes apoptosis by inducing the release of proapoptotic factors from mitochondria, which ultimately leads to depolarization and ATP depletion (Kiechle \& Zhang, 2002). $\mathrm{H}_{2} \mathrm{O}_{2}$ is a potent oxidant that very rapidly depolarizes the mitochondrial membrane and causes oncotic death. Mitochondrial injury is common to both modes of death, and hence may be involved in both $C$. jejuni-induced apoptosis of macrophages (Siegesmund et al., 2004) and oncosis of enterocytes (this study). Importantly, mitochondrial injury may induce apoptosis if cellular ATP levels remain sufficient to complete early-stage ATP-dependent apoptotic events, but may induce oncosis if mitochondrial damage causes rapid and excessive ATP depletion (Eguchi et al., 1997; Leist et al., 1997). Thus, while ATP depletion may be evident in both apoptosis and oncosis, only oncosis arises as a consequence of ATP depletion (Kim et al., 2003). Enterocytes are relatively active metabolically (Ma et al., 2006), and thus may be particularly susceptible to the ATPdepleting effects of $C$. jejuni-induced mitochondrial injury and therefore exhibit rapid oncotic death. Interestingly, other enteric pathogens, including Shigella flexneri and pathogenic E. coli, also induce either oncosis or apoptosis depending on the cell line that is used (Fernandez-Prada et al., 1998; Nonaka et al., 2003).

The intrinsic apoptotic pathway is the best-characterized mechanism of mitochondria-dependent cell death. Activation of this pathway results in the formation of the apoptosome, which activates caspase- 9 and the apoptotic death cascade ( $\mathrm{Li}$ et al., 1997). Results from our study demonstrate that $C$. jejuni induces enterocyte death via a caspase-independent mechanism. Intriguingly, caspase-9independent mitochondrial dysfunction has also been implicated in C. jejuni-induced macrophage apoptosis, indicating that $C$. jejuni causes cell death via a unique mechanism that does not involve formation of the apoptosome (Siegesmund et al., 2004). We are currently determining whether enterocyte death is mitochondria dependent and identifying the specific mediators involved.

During host infection, enterocytes are among the first cells that $C$. jejuni interacts with. The abilities of $C$. jejuni to invade and/or kill host cells via cytotoxins are thought to be the primary factors responsible for disease (Ketley, 1997; Wassenaar \& Blaser, 1999). Interestingly, our findings indicate that $C$. jejuni invades enterocytes and causes oncosis by a mechanism that is independent of CDT, its only known toxin. The rapid nature of oncosis is not consistent with the slow apoptotic death typically associated with CDT intoxication (Purdy et al., 2000; Whitehouse et al., 1998). Also, we observed that enterocytes infected with the CDT mutant were killed to the same extent as those infected with wild-type C. jejuni. Most Campylobacter strains produce CDT (Bang et al., 2001; Eyigor et al., 1999; Pickett et al., 1996), and we verified the presence of the $c d t B$ gene in all but one of the clinical isolates that we examined. Consistent with a CDT-independent mechanism, oncosis was induced in a strain-dependent manner despite the ubiquitous presence of the $c d t B$ gene. Previous studies have shown that $C$. jejuni is able to induce monocyte and lymphocyte apoptosis in a CDT-independent fashion (Siegesmund et al., 2004; Zhu et al., 1999). It is possible that C. jejuni produces other cytotoxins besides CDT, including shiga-like toxin and haemolysin (Wassenaar, 1997), although this is controversial, as it is not supported by genome sequence analysis of $C$. jejuni strains. Regardless of the factors involved, the results presented here establish that $C$. jejuni causes CDTindependent oncosis.

Previous studies have suggested that cell death may be associated with invasion. Loss of epithelial cell viability has been shown to coincide with Campylobacter invasion (Newell \& Pearson, 1984). Furthermore, CDT-independent apoptosis was significantly reduced $(\sim 50 \%)$ in monocytes inoculated with an invasion-defective mutant (Siegesmund et al., 2004). Similarly, we observed that the ability to cause oncosis was significantly decreased in a FlaAFlaB mutant $\left(\mathrm{CDT}^{+}\right)$that was defective in the ability to adhere to and invade enterocytes, suggesting that association with host cells (i.e. adherence and/or invasion) is required to induce cytotoxic effects. Our results also indicated that clinical isolates of $C$. jejuni induced enterocyte oncosis in a strainspecific manner, and that cytotoxicity was correlated with invasion. Lack of standardized assays for assessing cytotoxicity and invasion makes comparisons among reports difficult. Additionally, many of the studies predate the discovery of the CDT, so it is not always clear whether cytotoxicity was confounded by the CDT. Despite these difficulties, our observations are in agreement with previous studies by Klipstein et al. $(1985,1986)$, in which cytotoxicity was found principally among highly invasive clinical isolates. Noteworthy is their observation that strains isolated from patients in whom disease was severe also exhibited higher levels of invasiveness and cytotoxicity than those from patients with less severe enteritis. The lack of patient data for the clinical isolates that we used prevented us from verifying these observations, but other studies have failed to establish an association between cytotoxicity and invasion (Lindblom \& Kaijser, 1995; Nadeau et al., 2003), possibly reflecting the variable nature of $C$. jejuni strains and/or different methodologies used for assessment of cytotoxicity. Further investigation into the causal relationship between enterocyte invasion and oncosis by $C$. jejuni and the relevance to clinical disease is warranted.

In summary, findings from our study demonstrate that $C$. jejuni induces rapid oncotic, rather than apoptotic, 
enterocyte death. Our findings also demonstrate that oncosis is CDT independent, requires $C$. jejuni-enterocyte association, is dependent on the strain of C. jejuni and correlates with increased invasion ability. These observations offer insight into the pathogenesis of this important enteric pathogen, as enterocyte oncosis and subsequent epithelial malfunction may contribute to the intense inflammation often associated with campylobacteriosis. Future investigations of $C$. jejuni-enterocyte interaction will elucidate the molecular mechanisms of C. jejuniinduced oncosis and assess its contribution to Campylobacter-induced enteritis.

\section{ACKNOWLEDGEMENTS}

We thank Byron Lee [Agriculture and Agri-Food Canada (AAFC), Lethbridge] for providing image analysis expertise. We also thank Kathaleen House and Jenny Gusse (AAFC, Lethbridge) for isolation and identification of clinical isolates. L.D. K. is supported by a studentship from the Food Safety and Food Quality National Program of AAFC. Campylobacter strain 81-176 was kindly provided by S. Logan (National Research Council, Ottawa, Canada). This study was supported by NSERC, the Crohn's and Colitis Foundation of Canada, and the Canada-Alberta Beef Industry Development Fund (CABIDF).

\section{REFERENCES}

Bang, D. D., Scheutz, F., Ahrens, P., Pedersen, K., Blom, J. \& Madsen, M. (2001). Prevalence of cytolethal distending toxin $(c d t)$ genes and CDT production in Campylobacter spp. isolated from Danish broilers. J Med Microbiol 50, 1087-1094.

Bielaszewska, M., Sinha, B., Kuczius, T. \& Karch, H. (2005). Cytolethal distending toxin from Shiga toxin-producing Escherichia coli $\mathrm{O} 157$ causes irreversible G2/M arrest, inhibition of proliferation, and death of human endothelial cells. Infect Immun 73, 552-562.

Blanke, S. R. (2005). Micro-managing the executioner: pathogen targeting of mitochondria. Trends Microbiol 13, 64-71.

Brennan, M. A. \& Cookson, B. T. (2000). Salmonella induces macrophage death by caspase-1-dependent necrosis. Mol Microbiol 38, 31-40.

Buret, A. G., Chin, A. C. \& Scott, K. G. (2003). Infection of human and bovine epithelial cells with Cryptosporidium andersoni induces apoptosis and disrupts tight junctional ZO-1: effects of epidermal growth factor. Int J Parasitol 33, 1363-1371.

Chin, A. C., Teoh, D. A., Scott, K. G., Meddings, J. B., Macnaughton, W. K. \& Buret, A. G. (2002). Strain-dependent induction of enterocyte apoptosis by Giardia lamblia disrupts epithelial barrier function in a caspase-3-dependent manner. Infect Immun 70, 3673-3680.

Chin, A. C., Vergnolle, N., MacNaughton, W. K., Wallace, J. L., Hollenberg, M. D. \& Buret, A. G. (2003). Proteinase-activated receptor 1 activation induces epithelial apoptosis and increases intestinal permeability. Proc Natl Acad Sci U S A 100, 11104-11109.

De Melo, M. A. \& Pechere, J. C. (1988). Effect of mucin on Campylobacter jejuni association and invasion on HEp-2 cells. Microb Pathog 5, 71-76.

De Melo, M. A., Gabbiani, G. \& Pechere, J. C. (1989). Cellular events and intracellular survival of Campylobacter jejuni during infection of HEp-2 cells. Infect Immun 57, 2214-2222.

Denis, M., Soumet, C., Rivoal, K., Ermel, G., Blivet, D., Salvat, G. \& Colin, P. (1999). Development of a m-PCR assay for simultaneous identification of Campylobacter jejuni and C. coli. Lett Appl Microbiol 29, 406-410.

Dong, Z., Saikumar, P., Weinberg, J. M. \& Venkatachalam, M. A. (1997). Internucleosomal DNA cleavage triggered by plasma membrane damage during necrotic cell death. Involvement of serine but not cysteine proteases. Am J Pathol 151, 1205-1213.

Earnshaw, W. C., Martins, L. M. \& Kaufmann, S. H. (1999). Mammalian caspases: structure, activation, substrates, and functions during apoptosis. Annu Rev Biochem 68, 383-424.

Eguchi, Y., Shimizu, S. \& Tsujimoto, Y. (1997). Intracellular ATP levels determine cell death fate by apoptosis or necrosis. Cancer Res 57, 1835-1840.

Enari, M., Sakahira, H., Yokoyama, H., Okawa, K., Iwamatsu, A. \& Nagata, S. (1998). A caspase-activated DNase that degrades DNA during apoptosis, and its inhibitor ICAD. Nature 391, 43-50.

Everest, P. H., Goossens, H., Butzler, J. P., Lloyd, D., Knutton, S., Ketley, J. M. \& Williams, P. H. (1992). Differentiated Caco-2 cells as a model for enteric invasion by Campylobacter jejuni and C. coli. J Med Microbiol 37, 319-325.

Eyigor, A., Dawson, K. A., Langlois, B. E. \& Pickett, C. L. (1999). Detection of cytolethal distending toxin activity and cdt genes in Campylobacter spp. isolated from chicken carcasses. Appl Environ Microbiol 65, 1501-1505.

Fernandez-Prada, C., Tall, B. D., Elliott, S. E., Hoover, D. L., Nataro, J. P. \& Venkatesan, M. M. (1998). Hemolysin-positive enteroaggregative and cell-detaching Escherichia coli strains cause oncosis of human monocyte-derived macrophages and apoptosis of murine J774 cells. Infect Immun 66, 3918-3924.

Fiorentini, C., Fabbri, A., Falzano, L., Fattorossi, A., Matarrese, P., Rivabene, R. \& Donelli, G. (1998). Clostridium difficile toxin B induces apoptosis in intestinal cultured cells. Infect Immun 66, 2660-2665.

Gao, J. X., Ma, B. L., Xie, Y. L. \& Huang, D. S. (1991). Electron microscopic appearance of the chronic Campylobacter jejuni enteritis of mice. Chin Med J (Engl) 104, 1005-1010.

Gaynor, E. C., Cawthraw, S., Manning, G., MacKichan, J. K., Falkow, S. \& Newell, D. G. (2004). The genome-sequenced variant of Campylobacter jejuni NCTC 11168 and the original clonal clinical isolate differ markedly in colonization, gene expression, and virulenceassociated phenotypes. J Bacteriol 186, 503-517.

Golstein, P. \& Kroemer, G. (2007). Cell death by necrosis: towards a molecular definition. Trends Biochem Sci 32, 37-43.

Hickey, T. E., Majam, G. \& Guerry, P. (2005). Intracellular survival of Campylobacter jejuni in human monocytic cells and induction of apoptotic death by cytholethal distending toxin. Infect Immun 73, 5194-5197.

Humphrey, C. D., Montag, D. M. \& Pittman, F. E. (1986). Morphologic observations of experimental Campylobacter jejuni infection in the hamster intestinal tract. Am J Pathol 122, 152-159.

Ketley, J. M. (1997). Pathogenesis of enteric infection by Campylobacter. Microbiology 143, 5-21.

Kiechle, F. L. \& Zhang, X. (2002). Apoptosis: biochemical aspects and clinical implications. Clin Chim Acta 326, 27-45.

Kim, J. M., Eckmann, L., Savidge, T. C., Lowe, D. C., Witthoft, T. \& Kagnoff, M. F. (1998). Apoptosis of human intestinal epithelial cells after bacterial invasion. J Clin Invest 102, 1815-1823.

Kim, J. S., He, L. \& Lemasters, J. J. (2003). Mitochondrial permeability transition: a common pathway to necrosis and apoptosis. Biochem Biophys Res Commun 304, 463-470.

Klipstein, F. A., Engert, R. F., Short, H. \& Schenk, E. A. (1985). Pathogenic properties of Campylobacter jejuni: assay and correlation with clinical manifestations. Infect Immun 50, 43-49. 
Klipstein, F. A., Engert, R. F. \& Short, H. B. (1986). Enzyme-linked immunosorbent assays for virulence properties of Campylobacter jejuni clinical isolates. J Clin Microbiol 23, 1039-1043.

Konkel, M. E., Klena, J. D., Rivera-Amill, V., Monteville, M. R., Biswas, D., Raphael, B. \& Mickelson, J. (2004). Secretion of virulence proteins from Campylobacter jejuni is dependent on a functional flagellar export apparatus. J Bacteriol 186, 3296-3303.

Korlath, J. A., Osterholm, M. T., Judy, L. A., Forfang, J. C. \& Robinson, R. A. (1985). A point-source outbreak of campylobacteriosis associated with consumption of raw milk. J Infect Dis 152, 592-596.

Lara-Tejero, M. \& Galan, J. E. (2001). CdtA, CdtB, and CdtC form a tripartite complex that is required for cytolethal distending toxin activity. Infect Immun 69, 4358-4365.

Leist, M., Single, B., Castoldi, A. F., Kuhnle, S. \& Nicotera, P. (1997). Intracellular adenosine triphosphate (ATP) concentration: a switch in the decision between apoptosis and necrosis. J Exp Med 185, 1481-1486.

Li, P., Nijhawan, D., Budihardjo, I., Srinivasula, S. M., Ahmad, M., Alnemri, E. S. \& Wang, X. (1997). Cytochrome $c$ and dATP-dependent formation of Apaf-1/caspase-9 complex initiates an apoptotic protease cascade. Cell 91, 479-489.

Lindblom, G. B. \& Kaijser, B. (1995). In vitro studies of Campylobacter jejuni/coli strains from hens and humans regarding adherence, invasiveness, and toxigenicity. Avian Dis 39, 718-722.

Los, M., Mozoluk, M., Ferrari, D., Stepczynska, A., Stroh, C., Renz, A., Herceg, Z., Wang, Z. Q. \& Schulze-Osthoff, K. (2002). Activation and caspase-mediated inhibition of PARP: a molecular switch between fibroblast necrosis and apoptosis in death receptor signaling. Mol Biol Cell 13, 978-988.

Ma, C., Wickham, M. E., Guttman, J. A., Deng, W., Walker, J., Madsen, K. L., Jacobson, K., Vogl, W. A., Finlay, B. B. \& Vallance, B. A. (2006). Citrobacter rodentium infection causes both mitochondrial dysfunction and intestinal epithelial barrier disruption in vivo: role of mitochondrial associated protein (Map). Cell Microbiol 8, 1669-1686.

Majno, G. \& Joris, I. (1995). Apoptosis, oncosis, and necrosis. An overview of cell death. Am J Pathol 146, 3-15.

Miller, J. F., Dower, W. J. \& Tompkins, L. S. (1988). High-voltage electroporation of bacteria: genetic transformation of Campylobacter jejuni with plasmid DNA. Proc Natl Acad Sci U S A 85, 856-860.

Monteville, M. R., Yoon, J. E. \& Konkel, M. E. (2003). Maximal adherence and invasion of INT 407 cells by Campylobacter jejuni requires the CadF outer-membrane protein and microfilament reorganization. Microbiology 149, 153-165.

Nadeau, E., Messier, S. \& Quessy, S. (2003). Comparison of Campylobacter isolates from poultry and humans: association between in vitro virulence properties, biotypes, and pulsed-field gel electrophoresis clusters. Appl Environ Microbiol 69, 6316-6320.

Newell, D. G. \& Pearson, A. (1984). The invasion of epithelial cell lines and the intestinal epithelium of infant mice by Campylobacter jejuni/coli. J Diarrhoeal Dis Res 2, 19-26.
Nonaka, T., Kuwabara, T., Mimuro, H., Kuwae, A. \& Imajoh-Ohmi, S. (2003). Shigella-induced necrosis and apoptosis of U937 cells and J774 macrophages. Microbiology 149, 2513-2527.

Palomba, L., Sestili, P., Columbaro, M., Falcieri, E. \& Cantoni, O. (1999). Apoptosis and necrosis following exposure of U937 cells to increasing concentrations of hydrogen peroxide: the effect of the poly(ADP-ribose)polymerase inhibitor 3-aminobenzamide. Biochem Pharmacol 58, 1743-1750.

Pickett, C. L., Pesci, E. C., Cottle, D. L., Russell, G., Erdem, A. N. \& Zeytin, H. (1996). Prevalence of cytolethal distending toxin production in Campylobacter jejuni and relatedness of Campylobacter sp. $c d t B$ gene. Infect Immun 64, 2070-2078.

Purdy, D., Buswell, C. M., Hodgson, A. E., McAlpine, K., Henderson, I. \& Leach, S. A. (2000). Characterisation of cytolethal distending toxin (CDT) mutants of Campylobacter jejuni. J Med Microbiol 49, 473-479.

Ren, Y. \& Savill, J. (1998). Apoptosis: the importance of being eaten. Cell Death Differ 5, 563-568.

Russell, R. G., O’Donnoghue, M., Blake, D. C., Jr, Zulty, J. \& DeTolla, L. J. (1993). Early colonic damage and invasion of Campylobacter jejuni in experimentally challenged infant Macaca mulatta. J Infect Dis 168, 210-215.

Siegesmund, A. M., Konkel, M. E., Klena, J. D. \& Mixter, P. F. (2004). Campylobacter jejuni infection of differentiated THP-1 macrophages results in interleukin 1 beta release and caspase-1-independent apoptosis. Microbiology 150, 561-569.

Smiley, S. T., Reers, M., Mottola-Hartshorn, C., Lin, M., Chen, A., Smith, T. W., Steele, G. D., Jr \& Chen, L. B. (1991). Intracellular heterogeneity in mitochondrial membrane potentials revealed by a Jaggregate-forming lipophilic cation JC-1. Proc Natl Acad Sci U S A 88, 3671-3675.

Stanfield, J. T., McCardell, B. A. \& Madden, J. M. (1987). Campylobacter diarrhea in an adult mouse model. Microb Pathog 3, 155-165.

Vandenabeele, P., Vanden Berghe, T. \& Festjens, N. (2006). Caspase inhibitors promote alternative cell death pathways. Sci STKE 2006, pe44.

Wassenaar, T. M. (1997). Toxin production by Campylobacter spp. Clin Microbiol Rev 10, 466-476.

Wassenaar, T. M. \& Blaser, M. J. (1999). Pathophysiology of Campylobacter jejuni infections of humans. Microbes Infect 1, 1023-1033.

Whitehouse, C. A., Balbo, P. B., Pesci, E. C., Cottle, D. L., Mirabito, P. M. \& Pickett, C. L. (1998). Campylobacter jejuni cytolethal distending toxin causes a G2-phase cell cycle block. Infect Immun 66, 1934-1940.

Zhu, J., Meinersmann, R. J., Hiett, K. L. \& Evans, D. L. (1999). Apoptotic effect of outer-membrane proteins from Campylobacter jejuni on chicken lymphocytes. Curr Microbiol 38, 244-249.

Zychlinsky, A. (1993). Programmed cell death in infectious diseases. Trends Microbiol 1, 114-117.

Edited by: P. W. O'Toole 